\title{
Comparison of Quantitative EEG Indices of the Patients with Alzheimer and Parkinson
}

\author{
Seyed Mahmoud Tabatabaei* \\ Department of Physiology, Tabriz Branch, Islamic Azad University, Tabriz, Iran.
}

*Corresponding author: Seyed Mahmoud Tabatabaei, Department of Physiology, Tabriz Branch, Islamic Azad University, Tabriz, Iran.

ARTICLE INFO

Received: 蔧 August 15, 2019

Published: 蔧 August 26, 2019

Citation: Seyed Mahmoud Tabatabaei. Comparison of Quantitative EEG Indices of the Patients with Alzheimer and Parkinson. Biomed J Sci \& Tech Res 20(5)-2019. BJSTR. MS.ID.003520

Keywords: Alzheimer Disease; Parkinson's disease; Absolute power; Coherence and QEEG.

\section{ABSTRACT}

Background and Aims: The two common age-related neurological Alzheimer's Disease (AD) and Parkinson's disease Dementia (PDD) both involve the people with behavioral and cognitive problems. The brain waves of these people change and affect their brain functions.

Methods: Forty patients suffering from Alzheimer Disease and 12 patients with Parkinson Dementia and 40 healthy subjects were included in the study. The mean absolute power and mean coherence of brain waves were studied using brain waves analysis with QEEG method.

Results: The results showed that mean absolute power of Delta and Theta waves in PDD subjects was higher than those of the AD and control groups, but the absolute power mean of Alpha in Parkinson Dementia patients was lower than that of the Alzheimer and control group; however, the difference between the two dementia groups in terms of Beta absolute power was not significant $(\mathrm{P}>0.05)$. There is significant difference between Alpha and Beta bands of the two hemispheres in frontal areas' coherence (F3$\mathrm{F} 4)(\mathrm{P}<0.05)$, but there is no significant difference in the other areas.

Conclusion: QEEG technique can be used as an accurate tool in timely diagnosis of $\mathrm{AD}$ and PDD diseases through evaluating the quantitative indices of brain waves.

\section{Introduction}

Two common age-related neurological Alzheimer Disease (AD) and Parkinson's disease Dementia (PDD) both affect the people and have severe psychological consequences. The main characteristic of $\mathrm{AD}$ is dementia, but the characteristics of PD include movement problems and cognitive disorders and dementia that is known as Parkinson's disease dementia is observed among 30\% of these patients [1]. Alzheimer disease (AD) is a disorder in brain function in which the mental power of the patient gradually weakens. The most obvious type of dementia is memory disorder. Mental disorder is generated gradually and it later progresses. One of the main reasons of reduced cognitive and behavioral functions in higher ages is the dementia that occurs besides cognitive and behavioral disorders among the elderly people. Alzheimer is the commonly identified reason for dementia. Clinical signs of the illness include memory disorders, speech and language abnormalities, as well as functional and executive impairments.
Parkinson diseases happen due to the lack of progressive neurons responsible for Dopamine neurotransmitter in Substantia nigrea of the brain. No treatment has been identified for preventing dementia in patients with PPD. Therefore, it is vital to find early diagnosis methods for diagnosing the symptoms of the disease. As the nature of both diseases show, studying brain processes in these patients is very important. One of the important activities of the brain is electrical activity that is studied in the recorded form of the brain waves using EEG (Electroencephalography). EEG analysis is considered as a useful method in the diagnosis of both diseases [2]. Until recently, the studies on brain electrophysiology were limited to the morphologic changes of brain waves in different diseases. But progresses in neuroscience led to quantification of brain electrical functions.

Most researchers have used QEEG (Quantitative Electroencephalography) in order to show the efficiency between 
dementia and increased bands with slower frequencies and reduced bands with higher frequencies in people with PD [3]. The most common QEEG findings represent replacement of background frequency to Delta and Theta waves and reduced central Alpha wave in patients with Alzheimer disease [4]. Sandmann et al. [5] observed direct relationship between severity of cognitive disorders and lower power of brain's electric frequency in Alzheimer patients [5]. Other studies have shown that the indicators of brain electrical functions among the elderly people show reduced power in different bands; these changes are mostly observed on Occipital, Parietal and Temporal lobes [6,7]. Fluctuations in low power of brain waves are observed not only in the elderly people, but they are also observed in the patients with Alzheimer disorder.

As it was mentioned, the patients' brain electrical function is determined by higher power of Delta and Theta waves and reduced power of Alpha and Beta waves [8,9]. These abnormalities in the electrical function of brain happen along with the changes in brain cortical blood flow and its metabolism [10]. Increased functions of slow waves are reported for Parkinson's dementia in quantitative electroencephalography [11]. Although, there is no problem in clinical diagnosis of between Alzheimer and Parkinson diseases, but understanding different pathophysiological mechanisms of both diseases in terms of prognosis can be important. This paper`s aim is to study the brain's electrical functions in different bands of different brain lobes and tries to evaluate the absolute power and coherence value of brain waves in patients with Alzheimer Disease and Parkinson dementia.

\section{Materials and Methods}

In a comparative casual study, 40 patients with Alzheimer, 12 patients with Parkinson Dementia and 40 healthy people, were included in a study by convenience sampling method. MMSE test was completed for all patients and healthy people. Then, EEG of

Table 1: General demographic data of patients and the control group.

\begin{tabular}{|c|c|c|c|c|c|}
\hline Groups & AD & PD & Control & $\mathbf{F}$ & Compare with ( $p$ Value) \\
\hline Number & 40 & 12 & 40 & - & - \\
\hline Age (year) & $74.7 \pm 8$ & $70.3 \pm 6$ & $69.8 \pm 2$ & 4.49 & ANOVA $(0.005)$ \\
\hline Sex (Male/Female) & $16.22 \pm 6$ & $4.8 \pm 9$ & $14.23 \pm 1$ & - & Chi- square $(0.176)$ \\
\hline Education (Year) & 3 & 4 & 5 & 2.13 & ANOVA (0.137) \\
\hline MMSE Test & 15.8 & 12.5 & 25.7 & 65.12 & ANOVA $(0.0001)$ \\
\hline
\end{tabular}

Table 2: Comparison of absolute power of brain waves in different groups.

\begin{tabular}{|c|c|c|c|c|c|c|c|c|}
\hline Mean & AD & PDD & Control & F & ANOVA (P Value) & PDD with AD & PDD with Control & AD with Control \\
\hline Delta & 33.7 & 37.3 & 29.7 & 6.91 & 0 & S & S & S \\
\hline Theta & 38.9 & 47 & 33.3 & 8.66 & 0 & S & S & S \\
\hline Alpha & 43.6 & 38.6 & 46.2 & 0.08 & 0 & S & S & S \\
\hline Beta & 29.5 & 31.3 & 35.2 & 0.65 & 0.58 & NS & S & S \\
\hline
\end{tabular}

S: Significant

NS: Non-Significant the patients and the subjects in the control group was obtained using electrocardiogram Mitsar model in eyes close position for duration of $10 \mathrm{~min}$. The brain waves were recorded using electroencephalograph or EEG with 21 channel amplifiers, plastic caps, and aluminum electrodes as well as 10-20 standard placement system. Only the waves free from artifacts were analyzed. The absolute power was analyzed separately for each band and the coherence between the hemispheres was calculated using the following pairs: Left-right frontal (F3-F4); left-right temporal (T3T4) \& left-right Occipital (01-02). Then the data were analyzed using Neuroguid software. Frequency domain analysis was done using Fast Fourier Transform (FFT) algorithm. One-way ANOVA test was used in order to evaluate the mean absolute power of waves and to measure coherence between the hemispheres. MannWhitney U test was used for follow-up tests. In all cases, the values less than 0.05 were considered significant.

\section{Results}

General demographic values are provided in Table 1 . Table 1 shows that there is a difference between scores of MMSE between the groups (ANOVA, $\mathrm{p}<0.05$ ). MMSE score of patients was lower than that of the control group. But Duncan test shows that the difference between PD and AD is not significant. ANOVA test was used in order to examine the absolute power of brain waves in the patients with Alzheimer and Parkinson. The results are provided in Table 2. Mean of Delta and Theta absolute powers of PDD patients is higher than those of $\mathrm{AD}$ and control group; Alpha absolute power of Parkinson dementia is lower than that of the Alzheimer and control groups, while that in Alzheimer is lower than that of the control group. While there is significant difference between Alzheimer and Parkinson groups with control group in terms of Beta wave absolute power - especially high Beta- there is no significant differences between them compared to the two dementia groups (ANOVA, P> 0.05 ) (ANOVA and Duncan test, $\mathrm{p}<0.05$ ). 
One-way ANOVA was used in order to compare the coherences in the brain waves of the patients with Alzheimer and Parkinson diseases in both Frontal lobes ( F3 \& F4). The test data are provided in Table 3. There is significant difference between Alpha and Beta Table 3: The value of coherences between the left and right hemisphers of the frontal regions(F3-F4) and comparing in three group.

\begin{tabular}{|c|c|c|c|c|c|c|c|c|}
\hline Mean: & AD & PDD & Control & F & ANOVA (P Value) & PDD with AD & PDD with Control & AD with Control \\
\hline Delta & 0.56 & 0.61 & 0.59 & 0.88 & 0.646 & NS & NS & S 0.01 \\
\hline Theta & 0.54 & 0.63 & 0.57 & 3.44 & 0.039 & S 0.031 & NS & S 0.01 \\
\hline Alpha & 0.51 & 0.62 & 0.59 & 3.29 & 0.049 & S 0.001 & NS & S 0.011 \\
\hline Beta & 0.42 & 0.59 & 0.56 & 9.65 & 0 & S 0.025 & S 0.01 & S 0.001 \\
\hline
\end{tabular}

S: Significant

NS: Non-Significant

\section{Discussion}

Experimental evidences show that Delta, Theta and Alpha waves fluctuations represent the cognitive and memorial functions of humans and studying the changes in these waves may indicate variable types of disorders and psychiatric diseases. In this regard, Quantified techniques can increase the sensitivity of electroencephalography [12]. In Alzheimer disease and the activity of brain waves in these patients, EEG findings indicate increased Delta and Theta waves and reduced Alpha and Beta waves [4]. These findings are consistent with the findings of our study; although this group did not report anything on Beta wave. Our results related to increased Theta and Delta power in AD are similar to the results of Chiaramonti et al. [13]. However, reduced absolute power for Alpha and Beta waves was not reported in theirs, it may be due to Alzheimer disease at its weak stage [13].

The research of Fonseca et al. [12] on the absolute power of brain waves in Alzheimer and control group showed that Alzheimer patients have higher absolute power for Delta and Theta waves compared to the control group; furthermore, Beta and Alpha waves' coherence in frontal areas were lower than that of the control group [14]. These findings are consistent with the results of our study. It seems that activity of slow waves is more than that of Alpha and Beta waves' not only in Alzheimer but also in other states of dementia in which brain cells dementia are observed. Martin-Loeches reported wide distribution of slow brain rhythms in vascular dementia [15]. Increased absolute power of slow waves in PDD group is similar to the results obtained by Sandmann et al. [5], Sinanovic et al. [11], Fonseca and et al. [14]. Distributed changes are seen in slow frequency of Delta and Theta bands of AD and PDD groups. However, increased activity of slow waves in PDD was higher than that of $\mathrm{AD}$, and these results were similar to the results obtained by Babiloni et al. [16]. They showed more Theta/ Delta increase in PDD patients than AD patients.

The next issue about brain features is coherence. In other words, coherence measures covariance between pair signals or pair electrodes and it is a function of frequency; this method is good establishment of the connection between the two areas through bands of the two hemispheres in terms of coherence in the Frontal areas; it is higher for PDD group than AD group, and it is lower for AD group than the control group (Mann Whitney $U, p<0.05$ ). There is no significant difference in other areas of both hemispheres. 
brain electrical waves among the people with Alzheimer disease; this is considered as a means of diagnosing Alzheimer especially in its initial stages. Like our research, they also reported statistically significant difference in the coherence of frontal areas. It seems that due to the importance of high frequency waves in creating brain arousal states and reduced frontal areas in patients with dementia, these waves have reduced both Alzheimer and Parkinson's disease dementia in terms of power and necessary coherence compared to both hemispheres.

\section{Conclusion}

In recent decades, various methods and techniques have been developed and applied for the fastest, most available, noninvasive diagnosis of neurologic disease including Alzheimer and Parkinson's dementia. However, electroencephalography and its recent quantitative form or QEEG has gained special place in this regard. Maybe the main reason is application of FFT algorithm with the ability of providing more information about the characteristics of brain waves. Converting synovial waves into quantified waves, in addition to enabling comparison of these waves with those of the healthy people as well as creating brain maps, lead to easy and fast diagnosis of these diseases. Various components including absolute power of brain waves and coherence of waves make it feasible to evaluate the brain functions resulting from waves. As previous studies and current study show, the activity of slow frequency waves (Delta and Theta) in elderly people represents dementia disorders. Beta and Alpha waves of these patients show weak functions compared to healthy people. In the case of coherence, reduced isotonic of mirrored points in the areas between the two hemispheres can be observed due to degradation of cortex neuron cells especially in White matter area in terms of brain arousal waves (Beta and especially Alpha); this issue can be observed more clearly in Frontal areas.

\section{Acknowledgment}

The author thanks sincerely all participants and their families.

\section{References}

1. Aarsland D, Zaccai JCB, Brayne C (2005) A systematic review of prevalence studies of dementia in Parkinson's disease. Mov Disord 20(10): 1255-1263.

2. Luccas FJ, Anghinah R, Braga NI, Fonseca LC, Frochtengarten ML, et al (1999) Guidelines for recording/analyzing quantitative EEG and evoked potentials. Part II: Clinical aspects. Arq Neuropsiquiatr 57(1): 132-146.

3. Serizawa K, Kamei S, Morita A, Hara M, Mizutani T, et al. (2008) Comparison of quantitative EEGs between Parkinson disease and ageadjusted normal controls. J Clin Neurophysiol 25(6): 361-366.

4. Klass DW, Brenner RP (1995) Electroencephalography of the elderly. Journal of Clinical Neurophysiology 12(2): 116-131.

5. Sandmann M, Piana E, Sousa D, Bittencourt P (1996) Digital EEG with brain mapping in Alzheimer's dementia and Parkinson's disease. A prospective controlled study. Arq Neuropsiquiatr 54(1): 50-56.
6. Klimesch W (1999) EEG alpha and theta oscillations reflect cognitive and memory performance: A review and analysis. Brain Research Reviews 29(2-3): 169-195.

7. Babiloni C, Binetti G, Cassetta E, Dal Forno G, Del Percio, et al. (2006) Sources of cortical rhythms change as a function of cognitive impairment in pathological aging: A multi-centric study. Clinical Neurophysiology 117(2): 252-268.

8. Auwels J, Vialatte F, Musha T, Cichocki A (2010) A comparative study of synchrony measures for the early diagnosis of Alzheimer's disease based on EEG. Neuroimage 49(1): 668-693.

9. Babiloni C, Babiloni F, Carducci F, Cappa S, Cincotti F, et al. (2004) Human cortical rhythms during visual delayed choice reaction time tasks. highresolution EEG study on normal aging. Behavioral Brain Research 153(1): 261-271.

10. Rodriguez G, Copello F, Nobili F, Vitali P, Perego, G, et al. (1999) EEG spectral profile to stage Alzheimer's disease. Clinical Neurophysiology 110(10): 1831-1837.

11. Sinanovic 0, Kapidzic A, Kovacevic L, Hudic J, Smajilovic (2005) EEG frequency and cognitive dysfunction in patients with Parkinson's disease. Med Arh 59(5): 286-287.

12. Fonseca L, Tedrus G, Letro G, Bossoni A (2009) Dementia, mild cognitive impairment and quantitative EEG in patients with Parkinson's disease. Clin EEG Neurosci 40(3): 168-172.

13. Chiaramonti R, Muscas GC, Paganini M, Muller TJ, Fallgatter AJ, et al. (1997) Correlations of topographical EEG features with clinical severity in mild and moderate dementia of Alzheimer type. Neuropsychobiology 36(3): 153-158.

14. Fonseca LC, TedrusA S, Prandi LR, Andrade AC (2011) Quantitative electroencephalography power and coherence measurements in the diagnosis of mild and moderate Alzheimer's disease. Arq Neuropsiquiatr 69(2B): 297-303

15. Martin Loeches M, Garcia Trapero J, Gil P, Rubia FJ (2011) Topography of mobility and complexity parameters of the EEG in Alzheimer's disease. Biol Psychiatry 30(11): 1111-1121.

16. Babiloni C, Pandis M, Vecchio F, Buffo P, Sorpresi F, et al. (2011) sources of resting state electroencephalographic rhythms in Parkinson's disease related dementia and Alzheimer's disease. Clinical Neurophysiology 122(12): 2355-2364.

17. Nielsen TMJ, Lassonde M (1993) Decreased Interhemisferic EEG Coherene during sleep in agenesis of the corpus calosum. Eur Neurol 33(2): 173-176

18. Claus JJ, Strijers RL, Jonkman EJ, Ongerboer de Visser BW, Jonker C, et al. (1999) The diagnostic value of electroencephalography in mild senile Alzheimer's disease. Clin Neurophysiol 110(5): 825-832.

19. Besthorn C, Förstl H, Geiger Kabisch C, Sattel H, Gasser T, et al. (1994) EEG coherence in Alzheimer disease. Electroencephalogr Clin Neurophysiol $90(3): 242-245$.

20. Anghinah R, Kanda PA, Jorge MS, Lima EE, Pascuzzi L, et al. (2000) Alpha band coherence analysis of EEG in healthy adult's and Alzheimer's type dementia patients. Arq Neuropsiquiatr 58(2A): 272-275.

21. Adler G, Brassen S, Jajcevic J (2003) EEG coherence in Alzheimer's dementia. J Neural Transm 110(9): 1051-1058.

22. Locatelli T, Cursi M, Liberati D, Franceschi M, Comi G (1998) EEG coherence in Alzheimer's disease. Electroencephalogr Clin Neurophysiol 106(3): 229-237.

23. Kuks JB, Vos JE, O’Brien MJ (1987) Coherence patterns of the infant sleep EEG in absence of the corpus callosum. Electroencephalogr Clin Neurophysiol 66(1): 8-14. 
ISSN: 2574-1241

DOI: 10.26717/BJSTR.2019.20.003520

Seyed Mahmoud Tabatabaei. Biomed J Sci \& Tech Res

(C) This work is licensed under Creative

Submission Link: https://biomedres.us/submit-manuscript.php

$\begin{array}{ll}\text { BIOMEDICAL } & \text { Assets of Publishing with us } \\ \text { RESEARCHES } & \text { - Global archiving of articles } \\ & \text { - Immediate, unrestricted online access } \\ & \text { - Rigorous Peer Review Process } \\ \end{array}$

